\title{
Atropine 0.01\% Eye Drops for Myopia Control in American Children: A Multiethnic Sample Across Three US Sites
}

\author{
G. Luke Larkin - Alifiya Tahir - K. David Epley • Cynthia L. Beauchamp • \\ John T. Tong $\cdot$ Robert A. Clark (D)
}

Received: September 4, 2019 / Published online: October 10, 2019

(c) The Author(s) 2019

\begin{abstract}
Introduction: Topical atropine eye drops at low concentrations have been shown to slow myopia progression in East Asian studies. This study explored the effect of atropine $0.01 \%$ eye drops on controlling myopia progression in a multiethnic cohort of children in the USA.

Methods: A multicenter retrospective case-control study $(n=198)$ quantified the effect of adding nightly atropine $0.01 \%$ eye drops to treatment as usual on the progression
\end{abstract}

Enhanced Digital Features To view enhanced digital features for this article go to https://doi.org/10.6084/ m9.figshare.9901109.

G. L. Larkin

Northeast Ohio Medical University \& US Acute Care Solutions, 525 East Market Street, PO Box 2090,

Akron, OH 44309, USA

A. Tahir

Tisch MS Research Center of New York, 521 W 57th Street, New York, NY 10019, USA

K. D. Epley

Children's Eye Care, 11800 NE 128TH ST \#430,

Kirkland, WA 98034, USA

C. L. Beauchamp $\cdot$ J. T. Tong

ABC Eyes Pediatric Ophthalmology, PA, 7150

Greenville Ave, \#305, Dallas, TX 75231, USA

\section{R. A. Clark ( $\square)$}

Family Eye Medical Group, 4100 Long Beach Blvd, \#108, Long Beach, CA 90807, USA

e-mail: drraclark@yahoo.com of childhood (ages 6-15 years) myopia. Cases included all children treated with atropine for at least 1 year. Controls were matched to cases on both age ( \pm 6 months) and baseline spherical equivalent refraction (SER) ( \pm 0.50 diopters, D) at treatment initiation. The primary endpoint was the average SER myopia progression after $1,1.5$, and 2 years of therapy. A secondary outcome was the percentage of subjects with a clinically significant worsening of myopia, defined as a greater than -0.75 D SER increase in myopia.

Results: The average baseline SERs for the atropine $(n=100)$ and control $(n=98)$ groups were similar $(-3.1 \pm 1.9 \mathrm{D}$ and $-2.8 \pm 1.6 \mathrm{D}$, respectively) $(p=0.23)$. The average SER increase from baseline was significantly less for the atropine group than the control group at year $1 \quad(-0.2 \pm 0.8 \mathrm{D} \quad$ compared with $-0.6 \pm 0.4 \mathrm{D}, \quad p<0.001)$ and at year 2 $(-0.3 \pm 1.1 \mathrm{D}$ compared with $-1.2 \pm 0.7 \mathrm{D}$, $p<0.001)$. Secondary analysis at year 2 revealed that $80 \%$ of the control group vs. $37 \%$ of the atropine group experienced clinically significant worsening myopia of at least $-0.75 \mathrm{D}$ $(p<0.001)$. There were no major safety issues reported in either group.

Conclusion: Similar to results reported in Asia, atropine $0.01 \%$ eye drops significantly reduced myopia progression in a cohort of US children over 2 years of treatment.

Funding: Nevakar, Inc. 
Plain Language Summary: Plain language summary available for this article.

Keywords: Atropine; Childhood myopia; Eye drops; Myopia; Refractive error; Side effects

\section{PLAIN LANGUAGE SUMMARY}

Childhood nearsightedness (myopia) is increasing in both incidence and severity. Given the association of myopia with many serious eye diseases, multiple techniques have been used to try to slow the progression of myopia-increasing sunlight exposure, nightly hard contact lenses to reshape the surface of the eye, bifocals to reduce near vision strain, and medicated eye drops. Atropine eye drops appear to have the strongest effect in reducing myopia, but can also dilate the pupil and blur near vision. In recent Asian studies, a very dilute concentration of atropine, $0.01 \%$, has been shown to have reasonable efficacy compared with the standard $0.5 \%$ or $1 \%$ concentrations in reducing myopic progression, but with minimal pupil dilation and no blurred near vision. This study examined the effects of atropine $0.01 \%$ on myopic progression in a multiethnic, geographically diverse US population to see if those results are applicable to other populations. Children ages 6-15 years with myopia were divided into cases that received nightly atropine $0.01 \%$ in addition to eyeglasses and controls who only received eyeglasses. After 1 year of treatment, the atropine-treated patients had only $1 / 3$ the myopic progression of the controls without atropine. After 2 years, only $37 \%$ of the atropine group had sufficient myopic progression to require new eyeglasses compared with $80 \%$ of the control group. Importantly, there were no significant adverse effects noted in the atropine group. These results demonstrate that atropine $0.01 \%$ eye drops can be effective in reducing myopic progression in an ethnically diverse US population.

\section{INTRODUCTION}

Myopia is a major public health concern with both increasing incidence and increasing severity in the USA $[1,2]$. In the USA, an estimated $42 \%$ of the population is myopic, up from $25 \%$ in the 1970 s [1]. Over $1 / 3$ of children will become myopic by adulthood [2-4] and that proportion appears to be growing at an alarming rate [5-7].

Childhood myopia typically presents between 6 and 12 years of age [3]. According to the American Academy of Ophthalmology (AAO), the mean rate of progression of primary myopia is $0.5 \mathrm{D} /$ year, based on studies of mostly Caucasian children [1]. For the increasing proportion of children with higher myopia (-6.0 D or worse), there is a concomitant increase in the risk of severe and irreversible loss of vision over time, including retinal detachment, subretinal neovascularization, early and dense cataracts, and glaucoma [4, 7]. The rapid increase in the prevalence and severity of myopia is becoming a worldwide public health issue; therefore, finding methods to reduce progression of myopia is becoming increasingly important in industrialized nations [7].

Recently it was demonstrated in a study of 400 myopic children from Singapore that daily atropine $0.01 \%$ eye drops reduced the annual increase in mean spherical equivalent of refraction (SER) compared to the placebo arm of a prior study $[8,9]$. Concentrations of topical atropine evaluated in multiple Asian trials have ranged from $1 \%$ to $0.01 \%$, but the $0.01 \%$ concentration had shown the most durable effect on stabilizing the SER, plus had the benefit of the lowest incidence of side effects and the least likelihood of regression following cessation of treatment.

The measure of refractive change in SER/year is accepted globally as a clinically relevant marker for the progression of myopia. The atropine for the treatment of myopia (ATOM) 1 study included children 6-12 years of age with myopia of -1.0 to $-6.0 \mathrm{D}$. Over a period of 12 and 24 months, the average $( \pm \mathrm{SD})$ change in SER for children in the placebo group was $-0.76 \pm 0.44 \mathrm{D}$ and $-1.20 \pm 0.69 \mathrm{D}$, 
respectively [9]. This negative change on placebo was significantly larger than that found in atropine-treated eyes in the follow-up ATOM2 study, which assessed atropine concentrations of $0.01 \%, 0.1 \%$, and $0.5 \%$ [8]. After 2 years of atropine therapy, the average progression was $-0.30 \pm 0.60, \quad-0.38 \pm 0.60, \quad$ and $-0.49 \pm 0.63 \mathrm{D}$ in the atropine $0.5 \%, 0.1 \%$, and $0.01 \%$ groups, respectively. These data indicate the $0.5 \%$ and $0.1 \%$ doses were slightly more effective than the $0.01 \%$ dose. However, when atropine dosing was stopped for 12 months after 2 years of treatment, there was a rebound with worsening myopia in children originally treated with the higher concentrations of the drug, whereas those that received $0.01 \%$ atropine had minimal $(-0.28 \mathrm{D})$ change with preservation of myopia reduction in $74 \%$ of subjects [10].

Since most studies with atropine for myopia were performed in ethnic Asian populations, it is not clear if the differences in the presentation and progression of myopia in other countries might be associated with a different response to low concentration atropine drops. One small, 32-subject, case-control study in a single US pediatric ophthalmologic practice suggested that atropine $0.01 \%$ significantly reduced the rate of myopic progression over 1 year with minimal side effects [11]. Another small, uncontrolled study in Germany detected minimal pupillary dilation and an insignificant reduction in accommodation $24 \mathrm{~h}$ after initiating treatment [12]. The current study was designed to amplify these prior data and to assess myopia progression in a much larger, multicenter, multiethnic group of children in the USA given nightly atropine $0.01 \%$ eye drops. The hypothesis of this study is that the mean SER increase in myopia over time from baseline would be less for atropine-treated children than for age- and refraction-matched controls.

\section{METHODS}

\section{Study Design}

The study protocol and investigators were approved by IRB Company, Inc., a central institutional review board (IRB), that granted a waiver for informed consent for this retrospective chart review. The research also conformed to the principles of the Declaration of Helsinki and all data collection was compliant with the US Health Insurance Portability and Accountability Act.

This multicenter, case-control, retrospective study was designed to examine the magnitude of myopic progression in American children who were prescribed a privately compounded solution of atropine $0.01 \%$ eye drops compared to controls that were treated as usual without atropine for a period of at least 1 year (12 \pm 2 months). Double-blinded physicians masked to the final refractive outcome reviewed the charts. SER was measured at baseline and at the 1-year follow-up visit; if available, SER values at 1.5 ( 1 year 2 months to 1 year 8 months) and 2 years (1 year 9 months to 2 year 5 months) were also collected. Data on demographics (gender, date of birth, ethnicity, and number of office visits), medical, and ocular treatment history were collected if available.

Charts documenting treatment with atropine $0.01 \%$ administered once daily at bedtime in both eyes were identified. The inclusion criteria for cases were ages at treatment outset from 6 to 15 years, baseline SER from -0.25 to $-8.0 \mathrm{D}$ in at least one eye, astigmatism (if present in either eye) less than or equal to $-2.0 \mathrm{D}$, and eye examinations spanning at least $12 \pm 2$ months of treatment. Cases with a medical history predisposing to severe myopia (e.g., Marfan syndrome, Stickler syndrome, retinopathy of prematurity), abnormal ocular refractive anatomy (e.g, . keratoconus, lenticonus, spherophakia), or previous intraocular or ocular laser surgery were excluded. Each case was age- and disease severity-matched to a control with initial myopia within $\pm 0.5 \mathrm{D}$ and age within \pm 6 months. 
Atropine $0.01 \%$ was manufactured from atropine $1 \%$ or from atropine powder by private compounding pharmacies in the USA. Myopia treatment as usual included prescribing either non-bifocal, non-progressive, single-vision corrective lenses or, for smaller SER myopia, no treatment. Treatment compliance was not assessed. Refraction was performed at all examinations with or without cycloplegia at the investigator's discretion, although the initial refraction was most often done after cycloplegia. The eye with the greater myopic SER was selected (or the right eye if both eyes had identical SER) for analysis.

\section{Outcome Measures}

The primary efficacy variable was progression of myopia, defined as the change from baseline SER, calculated as D/year, at $1,1.5$, and 2 years. For a secondary efficacy outcome, sensitivity analysis defined myopia progression of at least $-0.75 \mathrm{D}$ as a clinically significant worsening (based on expert opinion from the 2016 FDA Workshop on myopia progression [3]).

The safety profile of atropine $0.01 \%$ eye drops was assessed through review of treatment emergent adverse events (TEAEs) recorded in the charts. Any recorded medical finding that occurred after the administration of the study medication was considered a TEAE.

\section{Statistical Analyses}

The primary hypothesis was tested using a twosided paired $t$ test for the change in SER from baseline. A one-sample Wilcoxon rank sum test was also used to test the robustness of the above test. Summary statistics for continuous data included computations of the average, standard deviation, median, minimum, and maximum. Frequency distributions were carried out for discrete variables. All analyses were performed using SAS ${ }^{\circledR}$ Version 9.3. Results were considered statistically significant with a $P$ value less than 0.05. Approximately 100 cases and matched controls were included, giving the study 93\% power for detecting a difference of $0.30 \mathrm{D}$ between the treatment arms in change from baseline SER at the 5\% significance level using a standard deviation of $0.69 \mathrm{D}$ as demonstrated by Chia et al. [13].

\section{RESULTS}

There were 198 eligible charts (100 atropine and 98 controls) that qualified for the primary analysis at 1 year. Controls could not be identified for two cases, an Asian girl with baseline SER of $-8.0 \mathrm{D}$ at age 10 and a Caucasian girl with baseline SER of $-7.75 \mathrm{D}$ at age 11 . The average age at treatment onset was 9 years old (Table 1$)$ in both groups $(p=0.74)$. The atropine-treated subjects were primarily Asian (38\%) and Caucasian (35\%), with smaller percentages of mixed race $(7 \%)$, Native American (1\%), African American (3\%), and Hawaiian/ Pacific Islander (4\%). The ethnic background for $12 \%$ was not identifiable. The control subjects were also primarily Asian (10\%) and Caucasian (39\%), with smaller percentages of mixed race (2\%), Hawaiian/Pacific Islander (4\%), and African American (1\%). The ethnic background for $44 \%$ was not identifiable.

The average SERs at baseline for the atropine and control groups were not significantly different at $-3.1 \pm 1.9 \mathrm{D}$ and $-2.8 \pm 1.6 \mathrm{D}$, respectively $(p=0.23)$. In the atropine group, there was a trend for female patients $(-3.1 \pm 1.8 \mathrm{D})$ to be more myopic than male patients $(-2.8 \pm 1.9 \mathrm{D})(p=0.33)$. The baseline SERs were significantly higher among Asians compared to Caucasians $(-3.6 \pm 1.9 \mathrm{D}$ compared with $-2.5 \pm 1.7 \mathrm{D}, p=0.01)$. The ophthalmic history of the two groups was generally comparable (Table 1), except for a slightly higher prevalence of astigmatism in the atropine group ( $94 \%$ vs. $90 \%, p=0.27$ ).

The change from baseline SER for both groups is shown in Fig. 1. At year 1, the average change from baseline was $-0.2 \pm 0.8 \mathrm{D}$ in the atropine group and $-0.6 \pm 0.4 \mathrm{D}$ among controls, yielding an absolute difference of $-0.4 \mathrm{D}$ $(p<0.001)$. Only $49 \%$ of atropine-treated subjects and $56 \%$ of control subjects had an examination at year 2 . The average SER change from baseline was $-0.3 \pm 1.1 \mathrm{D}$ in the atropine group vs. $-1.2 \pm 0.7 \mathrm{D}$ among controls, 
Table 1 Summary by treatment group

\begin{tabular}{|c|c|c|c|}
\hline Characteristic/category & Statistic & Control $(N=98)$ & Atropine $(N=100)$ \\
\hline Baseline age (years) & Mean (SD) & $9.2(2.11)$ & $9.3(2.10)$ \\
\hline \multirow[t]{4}{*}{ Age groups $(N)$ (years) } & $N, 6$ to $<8$ & 42 & 42 \\
\hline & $N, 8$ to $<10$ & 32 & 31 \\
\hline & $N, 10$ to $<12$ & 15 & 18 \\
\hline & $N, 12$ to $<15$ & 9 & 9 \\
\hline \multicolumn{4}{|l|}{ Sex } \\
\hline Female & $N$ & 56 & 50 \\
\hline Male & $N$ & 42 & 50 \\
\hline \multicolumn{4}{|l|}{ Ophthalmic history } \\
\hline Myopia & $N(\%)$ & $98(100 \%)$ & $100(100 \%)$ \\
\hline Astigmatism & $N(\%)$ & $88(90 \%)$ & $94(94 \%)$ \\
\hline Exophoria & $N(\%)$ & $21(21 \%)$ & $21(21 \%)$ \\
\hline Amblyopia & $N(\%)$ & $6(6 \%)$ & $4(4 \%)$ \\
\hline Esophoria & $N(\%)$ & $6(6 \%)$ & $3(3 \%)$ \\
\hline Conjunctivitis & $N(\%)$ & $7(7 \%)$ & $3(3 \%)$ \\
\hline Baseline SER & Mean (SD) & $-2.8(1.6)$ & $-3.1(1.9)$ \\
\hline \multicolumn{4}{|c|}{ Change from baseline SER } \\
\hline \multirow[t]{3}{*}{ Year 1} & $N$ & 71 & 81 \\
\hline & Mean (SD) & $-0.6(0.4)$ & $-0.2(0.8)$ \\
\hline & Median & -0.5 & -0.3 \\
\hline \multirow[t]{3}{*}{ Year 1.5} & $N$ & 20 & 49 \\
\hline & Mean (SD) & $-0.9(0.6)$ & $-0.3(0.9)$ \\
\hline & Median & -0.8 & -0.3 \\
\hline \multirow[t]{3}{*}{ Year 2} & $N$ & 55 & 49 \\
\hline & Mean (SD) & $-1.2(0.7)$ & $-0.3(1.1)$ \\
\hline & Median & -1.3 & -0.3 \\
\hline
\end{tabular}

yielding an absolute difference of $-0.9 \mathrm{D}$ $(p<0.001)$ by the end of year 2 .

At year 1 , only $22 \%$ of atropine-treated subjects had worsening myopia greater than - $0.75 \mathrm{D}$ compared with $41 \%$ of controls $(p=0.004)$. At year 2 , both groups showed an increase in the proportion of those with worsening myopia greater than $-0.75 \mathrm{D}: 37 \%$ of atropine-treated subjects and $80 \%$ of control subjects demonstrated myopia progression greater than $-0.75 \mathrm{D}(p<0.001)$. The percentage of subjects with rapid worsening of myopia (SER > -1.0 D/year) was $4 \%$ and $11 \%$ for the atropine and control groups, respectively. The 


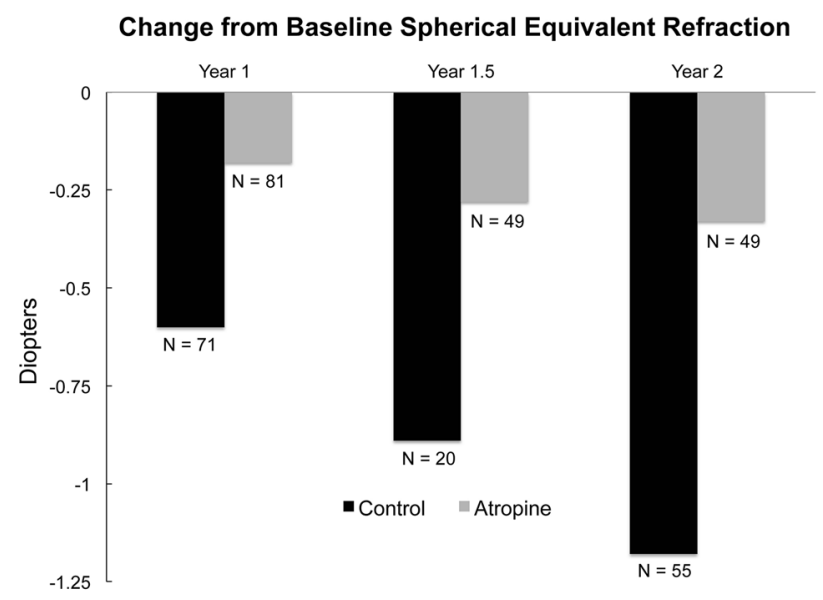

Fig. 1 Mean change from baseline in spherical equivalent refraction (SER) for each treatment group

percentage of subjects with minimal worsening or a decrease in myopia (SER $\leq-0.25 \mathrm{D} /$ year) was $57 \%$ and $15 \%$ for the atropine and control groups, respectively, after 2 years of follow-up.

Similar results were found for the percentage of subjects with a hyperopic shift, defined as an improvement in SER by at least $+0.25 \mathrm{D}$. After 1 year, $27 \%$ of atropine-treated subjects were found to have less myopia compared with $1 \%$ of controls. After 2 years, $22 \%$ of atropine-treated subjects still had less myopia than at treatment initiation compared with $2 \%$ of controls.

An exploratory analysis indicated that the change in SER between races after 1 year of treatment was comparable. Among Caucasians, the change from baseline SER in the atropine and control groups was from $-0.3 \pm 0.7$ to $-0.6 \pm 0.4 \mathrm{D}$, comparable to the SER change in Asians from $-0.3 \pm 0.7$ to $-0.8 \pm 0.4 \mathrm{D}$, respectively. Atropine-treated Caucasians and Asians both had less myopic progression, $-0.3 \mathrm{D}$ vs. $-0.5 \mathrm{D}$ respectively, compared to control patients.

Univariate analysis using age as a continuous variable demonstrated that the effect of atropine on stabilizing the baseline SER was consistent across all age groups, but the younger subjects had more myopic progression among both atropine-treated and control subjects. For example, the youngest age group of 6-8 years (42\% of the study population) progressed by $-0.3 \mathrm{D}$ in the atropine group compared with $-0.7 \mathrm{D}$ in the control group, a difference of
$0.4 \mathrm{D}(p=0.04)$, while the oldest age group of $13-15$ years (9\% of the study population) progressed by only $-0.3 \mathrm{D}$ in the control group while the atropine group actually regressed (improved) + 0.3 D, a difference of $0.6 \mathrm{D}$ $(p=0.007)$.

The number of subjects with at least one adverse event was $22(22 \%)$ in the atropine group and 27 (28\%) in the control group (Table 2). Some subjects had multiple symptoms. Sensitivity to light and mydriasis were reported in 1\% of atropine-treated patients and in no controls. Astigmatism, either novel or worsening, was reported in ten patients in the atropine group and 22 patients in the control group. Allergic conjunctivitis was noted in 3\% of the atropine group compared with none in the control group.

\section{DISCUSSION}

This study was a multicenter, case-control, retrospective chart review evaluating the realworld efficacy of atropine $0.01 \%$ eye drops for the treatment of progressive childhood myopia in a multiethnic population. Subjects in the control group received only typical eye care consisting of non-bifocal, non-progressive, single-vision lenses, or no treatment for low myopia. The use of single vision spectacles would not be expected to slow the progression of childhood myopia [5]. Compared to usual care, 
Table 2 Treatment emergent adverse events

\begin{tabular}{|c|c|c|c|}
\hline & Atropine & Control & Severity \\
\hline Allergy (redness and irritation) & 3 & 0 & Mild \\
\hline Anisometropia (OU) & 0 & 1 & Mild \\
\hline Astigmatism (novel or worsening) & 9 & 4 & Mild \\
\hline Astigmatism (novel or worsening) & 1 & 18 & Moderate \\
\hline Burning and itching $(\mathrm{OU})$ & 0 & 1 & Mild \\
\hline Corneal scratch & 1 & 0 & Mild \\
\hline Dizziness & 1 & 0 & Mild \\
\hline Enlarged cup to disc & 0 & 1 & Mild \\
\hline Dermatitis & 0 & 1 & Mild \\
\hline Esotropia surgery & 0 & 1 & Moderate \\
\hline Exophoria (OU) & 0 & 1 & Mild \\
\hline Exotropia & 1 & 0 & Mild \\
\hline Eye pain & 1 & 0 & Mild \\
\hline Floaters (OU) & 1 & 0 & Mild \\
\hline Headaches (non-ocular) & 0 & 1 & Mild \\
\hline Lupus & 0 & 1 & Mild \\
\hline Mild season allergy & 1 & 0 & Mild \\
\hline Mydriasis & 1 & 0 & Mild \\
\hline Myopia & 1 & 0 & Mild \\
\hline Posterior subcapsular cataracts & 0 & 1 & Mild \\
\hline Redness and irritation in both eyes & 1 & 0 & Mild \\
\hline Sensitivity to light & 1 & 0 & Mild \\
\hline Strabismus surgery $(\mathrm{OU})$ & 0 & 1 & Moderate \\
\hline Totals: events/subjects with events & $23 / 22$ & $32 / 27$ & \\
\hline
\end{tabular}

atropine-treated patients experienced a significant reduction in the average myopic progression of SER from baseline after 1, 1.5, and 2 years of treatment. These data suggest that atropine $0.01 \%$ could be an effective medication to slow childhood myopia and support the conduct of prospective, controlled clinical trials in populations other than the East Asian and Spanish populations studied previously $[8,14]$.
The data from this study in a US population are consistent with previous studies in Asian populations, which also demonstrated a beneficial effect of atropine $0.01 \%$ on childhood myopia progression [8]. The ATOM studies in Singaporean children showed a $0.33-\mathrm{D}$ average difference between $0.01 \%$ atropine and placebo after 1 year of treatment and a 0.71-D mean difference after 2 years. In our study, the mean differences between $0.01 \%$ atropine and usual 
care were $0.4 \mathrm{D}$ and $0.9 \mathrm{D}$ after 1 and 2 years, respectively. Most atropine-treated patients experienced slowing of myopic progression, with only $37 \%$ progressing more than $0.75 \mathrm{D}$ after 2 years. In contrast, $80 \%$ of controls experienced progression greater than $0.75 \mathrm{D}$ over 2 years. These results were both statistically significant and clinically meaningful, given that progression beyond $0.75 \mathrm{D}$ would indicate the need for a new spectacle prescription.

Atropine at higher concentrations than $0.01 \%$ has also been shown to slow myopic progression, but the real-world use of higher doses of atropine may be limited by side effects such as vision-related glare, photophobia, and near vision blur [15-17]. No safety concerns with atropine $0.01 \%$ treatment were apparent in our study. Most adverse events were mild and likely related to disease progression. Although atropine eye drops are used clinically to dilate the pupils, mydriasis and sensitivity to light were reported in only $1 \%$ of atropine-treated patients at this concentration. Treatment discontinuations were rare.

Despite a relatively small sample size, the impact of low-concentration atropine treatment was statistically significant $(p<0.001)$. However, there are several limitations to this study. First, it is a retrospective case-control study. It is nonetheless larger than other retrospective studies in North America to date. Multiple compounding pharmacies might introduce potential variance in drug stability, types of excipients, and compliance if not properly formulated for the ocular surface. Hyperopic shifts have been reported previously in ATOM studies in the $1.0 \%$ atropine group $[8,9]$, but this study reports hyperopic shifts at a much lower strength of atropine. This finding may reflect inconsistencies in the measurement of refraction, since these children did not consistently undergo cycloplegic refractions at all examinations. Finally, ethnicity data were not available for all of the patients. It is likely, however, that the population in this study reflects the racial and ethnic diversity of the participating US cities (Long Beach, California; Dallas/Fort Worth, Texas; and Kirkland, Washington). Interestingly, most of the children in this study are from the US Sun Belt, suggesting myopia in areas of higher ultraviolet B exposure may still benefit from topical low-concentration atropine [18].

The exact mechanism of action for atropine to reduce myopic progression is not currently known. Atropine has been shown to increase choroidal thickness in children [19], possibly through modulation of dopamine release [20], which has been correlated with a reduction in the rate of axial eye growth [21]. There are also scleral muscarinic receptors that might modulate the function of scleral fibroblasts and interfere with the scleral remodeling that accompanies progressive myopia [22]. The actual mechanism may include a combination of effects, but determination of the primary mechanism of action may allow more targeted therapy and/or alternative therapies for children who continue to demonstrate rapid myopic progression on treatment.

\section{CONCLUSION}

Data from this multiethnic retrospective study indicate that atropine $0.01 \%$ could be a welltolerated and effective pharmacologic intervention for slowing progression of childhood myopia. Four large multicenter prospective trials to confirm these observations are ongoing within the USA: the CHAMP (NCT03350620), STAAR (NCT03918915), MTSI (NCT03334253), and NCT03942419 trials.

\section{ACKNOWLEDGEMENTS}

Funding. Sponsorship for this study and the Rapid Service Fee were funded by Nevakar, Inc. WCCT Global, also funded by Nevakar, Inc., was responsible for data collection and statistical analysis after the cases were identified and matched with controls.

Authorship. All named authors meet the International Committee of Medical Journal Editors (ICMJE) criteria for authorship for this article, take responsibility for the integrity of 
the work as a whole, and have given their approval for this version to be published.

Disclosures. G. Luke Larkin was an employee of Nevakar, Inc. over the time period of the study and is now employed by Northeast Ohio Medical University \& US Acute Care Solutions. Robert A. Clark was a paid consultant for Nevakar, Inc., over the time period of the study. K. David Epley was a paid consultant for Nevakar, Inc., over the time period of the study. Cynthia L. Beauchamp was a paid consultant for Nevakar, Inc., over the time period of the study. Alifiya Tahir and John T. Tong have nothing to disclose.

Compliance with Ethics Guidelines. The study protocol and investigators were approved by IRB Company, Inc., a central IRB, that granted a waiver for informed consent for this retrospective chart review. The research also conformed to the principles of the Declaration of Helsinki and all data collection was compliant with the US Health Insurance Portability and Accountability Act.

Data Availability. All data generated or analyzed in this study are included in this published article.

Open Access. This article is distributed under the terms of the Creative Commons Attribution-NonCommercial 4.0 International License (http://creativecommons.org/licenses/ by-nc/4.0/), which permits any noncommercial use, distribution, and reproduction in any medium, provided you give appropriate credit to the original author(s) and the source, provide a link to the Creative Commons license, and indicate if changes were made.

\section{REFERENCES}

1. American Academy of Ophthalmology Refractive errors and surgery. Preferred Practice Patterns 2013. https://www.aao.org/preferred-practice-pattern/ refractive-errors-surgery-ppp-2013. Accessed 24 Jan 2018.
2. Holden BA, Fricke TR, Wilson DA, et al. Global prevalence of myopia and high myopia and temporal trends from 2000 through 2050. Ophthalmology. 2016;123:1036-42.

3. FDA Dermatologic and Ophthalmic Drugs Advisory Committee. Study Designs of Trials in the Treatment of Myopia. 2003. https://wayback.archiveit. org/7993/20170405133139/https://www.fda.gov/ ohrms/dockets/ac/03/briefing/3988B1_02_Novartis $\% 20$ Briefing\%20Document.pdf. Accessed 17 Jan 2017.

4. Foster PJ, Jiang Y. Epidemiology of myopia. Eye. 2014;28:202-8.

5. Gwiazda J, Hyman L, Hussein M, et al. A randomized clinical trial of progressive addition lenses vs. single vision lenses on the progression of myopia in children. Invest Ophthalmol Vis Sci. 2003;44:1492-500.

6. Vitale S, Sperduto RD, Ferris FL. Increased prevalence of myopia in the United States between 1971-1972 and 1999-2004. Arch Ophthalmol. 2009;127:1632-9.

7. Organization WH. The impact of myopia and high myopia: report of the Joint World Health Organization-Brien Holden Vision Institute Global Scientic Meeting on Myopia, University of New South Wales, Sydney, Australia, 16-18 March 2015. Geneva: World Health Organization; 2017.

8. Chia A, Chua WH, Cheung YB, et al. Atropine for the treatment of childhood myopia: safety and efficacy of $0.5 \%, 0.1 \%$, and $0.01 \%$ doses (atropine for the treatment of myopia 2). Ophthalmology. $2012 ; 119: 347-54$

9. Chua WH, Balakrishnan V, Chan YH, et al. Atropine for the treatment of childhood myopia. Ophthalmology. 2006;113:2285-91.

10. Chia A, Lu QS, Tan D. Five-year clinical trial on atropine for the treatment of myopia 2: myopia control with atropine $0.01 \%$ eyedrops. Ophthalmology. 2016;123:391-9.

11. Clark TY, Clark RA. Atropine $0.01 \%$ eye drops significantly reduce the progression of childhood myopia. J Ocul Pharmacol Ther. 2015;31:541-5.

12. Joachimsen L, Bohringer D, Gross NJ, et al. A pilot study on the efficacy and safety of $0.01 \%$ atropine in German schoolchildren with progressive myopia. Ophthalmol Ther. 2019;8:427-33.

13. Chia A, Chua WH, Wen L, Fong A, Goon YY, Tan D. Atropine for the treatment of childhood myopia: changes after stopping atropine $0.01 \%, 0.1 \%$ and 0.5\%. Am J Ophthalmol. 2014;157:451-7. 
14. Diaz-Llopis M, Pinazo-Duran M. Superdiluted atropine at $0.01 \%$ reduces progression in children and adolescents: a 5 year study of safety and effectiveness. Arch Soc Esp Oftalmol. 2018;93:182-5.

15. Walline JJ, Rah MJ, Jones LA. The children's overnight orthokeratology investigation (COOKI) pilot study. Optom Vis Sci. 2004;81:407-13.

16. Huang J, Wen D, Wang Q, et al. Efficacy comparison of 16 interventions for myopia control in children: a network meta-analysis. Ophthalmology. 2016;123:697-708.

17. Gong Q, Janowski M, Luo M, et al. Efficacy and adverse effects of atropine in childhood myopia: a meta-analysis. 2017;135:624-30. Ophthalmol.

18. Williams KM, Bentham GC, Young IS, et al. Association between myopia, ultraviolet $\mathrm{B}$ radiation exposure, serum vitamin D concentrations, and genetic polymorphisms in vitamin $\mathrm{D}$ pathways in a multicountry European study. JAMA Ophthalmol. 2017;135:47-53.

19. Zhang Z, Zhou Y, Zie Z, et al. The effect of topical atropine on the choroidal thickness of healthy children. Sci Rep. 2016;6:1-8.

20. Dhillon B, Armani J, Nickl L. The ocular growth inhibition effected by dopamine agonists and atropine is associated with transient increases in choroidal thickness in chicks. Invest Ophthalmol Vis Sci. 2008;49:1732.

21. Read SA, Alonso-Caneiro D, Vincent SJ, Collines MJ. Longitudinal changes in choroidal thickness and eye growth in childhood. Invest Ophthalmol Vis Sci. 2015;56:3103-12.

22. Barathi VA, Beuerman RG. Molecular mechanisms of muscarinic receptors in mouse scleral fibroblasts: prior to and after induction of experimental myopia with atropine treatment. Mol Vis. 2011;17:680-92. 